\title{
Estimates of the Laplacian Spectrum and Bounds of Topological Invariants for Riemannian Manifolds with Boundary II
}

\author{
Luca Sabatini
}

\begin{abstract}
We present some estimate of the Laplacian Spectrum and of Topological Invariants for Riemannian manifold with pinched sectional curvature and with non-empty and non-convex boundary with finite injectivity radius. These estimates do not depend directly on the the lower bound of the boundary injectivity radius but on the bounds of the curvatures of the manifold and its boundary.
\end{abstract}

\section{Introduction}

Estimates of the first non zero eigenvalue of the Laplace-Beltrami operator, named in what follows for the sake of simplicity the Laplacian, acting on a compact connected $n$-dimensional Riemannian manifold, have been calculated in the last decades of the XX Century by several authors. Payne and Weinberger, (1957) [7], Li and Yau, (1980) [5], Meyer, (1986) [6]) did estimate from below the first non zero eigenvalue as function of the diameter $\operatorname{diam}(M, g)$ of a compact connected Riemannian manifold of dimension $n$ and of a function $C$ depending only on the product of the lower bound $\delta$ of Ricci curvature of the manifold $\left(\right.$ Ricci $\left._{g} \geq-(n-1) \delta^{2} g\right)$ and the upper bound $D$ of its diameter. Cheng, (1975) [1], got upper bound of the Laplacian first non zero eigenvalue

Key Words: Double of a manifold; Laplacian; Topological invariants; Regularized metric with control of curvature.

2010 Mathematics Subject Classification: Primary 35P15 53C20; Secondary 53C21 $58 \mathrm{C} 40$.

Received: 22.03.2019.

Accepted: 26.07.2019 
ESTIMATES OF THE LAPLACIAN SPECTRUM AND BOUNDS OF TOPOLOGICAL INVARIANTS FOR RIEMANNIAN MANIFOLDS WITH BOUNDARY II.

of a compact connected $n$-dimensional Riemannian manifold as the sum of a part depending on the lower bound of Ricci curvature $\delta$ and a part depending on $\operatorname{diam}(M, g)$, via two constants $\Pi_{1}(n)$ and $\Pi_{2}(n)$ depending of the dimension $n$ only. Estimations are thus synthesized in the following chain of inequalities:

$$
\frac{C(D \cdot k)}{\operatorname{diam}^{2}(M, g)} \leq \lambda_{1}(M, g) \leq \Pi_{1}(n) \delta+\frac{\Pi_{2}(n)}{\operatorname{diam}^{2}(M, g)}
$$

Meyer shown also that, for manifolds with non-empty and non-convex boundary (see Definition 2.6), if the boundary injectivity radius $\operatorname{inj}_{\partial M}$ goes to zero, the Laplacian first non zero eigenvalue blows up. Estimates from below have been obtained by the writer in 2019 (see [8]), working on manifold with nonempty boundary and using the unitary approach of the double manifold. This method does allow to get a compact connected Riemannian manifold with empty boundary, the double manifold $(M \sharp M, g \sharp g)$, pasting two isometric copies of the same manifold $(M, \partial M, g)$ with non-empty boundary along their common boundary $\partial M$. The double manifold has a natural structure of $C^{\infty}$-manifold, however in a neighborhood of the gluing surface, the equator of the double manifold, the sectional curvature can reach negative values still high, this is the case of manifolds with non-convex boundary. This is the reason why estimates are not directly available if a finite lower bound of the curvature is required. To get them it is necessary to regularize the metric in a suitable neighborhood of the equator in such a way to obtain a new metric $\tilde{g}$, isometric to $g$ when restricted to each copy of the component manifolds and with the strong condition of the uniform control from below of the sectional curvature. Thanks to this regularization, estimates of the first eigenvalue of Laplacian are available and the blow up pointed out by Meyer is evident since the constants depending on the lower bound $a$ of the boundary injectivity radius go to infinity if $a \rightarrow 0^{+}$. In this paper, under the stronger assumptions " pinched sectional curvature of the manifold" $-k^{2} \leq \sigma \leq k^{2}$ and "pinched principal curvatures of the boundary " $-\eta \leq h_{\partial M} \leq \eta$ for some fixed positive numbers $k$ and $\eta$, we fix the lower and non zero bound $a$ of the boundary injectivity radius, getting lower and upper limitations of the new metric $\tilde{g}$ on $M \sharp M$, controlling the sectional curvature from below in a uniform manner. These bounds do not depend on the value of $a$. The following theorem collects the main functional results of this paper.

Main Theorem Let $(M, \partial M, g)$ be a compact connected n-dimensional Riemannian manifold with pinched sectional curvature for some positive real $k$, i.e. $-k^{2} \leq \sigma \leq k^{2}$, let $\partial M$ be its non-empty and non-convex boundary whose 
ESTIMATES OF THE LAPLACIAN SPECTRUM AND BOUNDS OF

TOPOLOGICAL INVARIANTS FOR RIEMANNIAN MANIFOLDS WITH

BOUNDARY II.

injectivity radius and main curvatures are such that

$$
\operatorname{inj}_{\partial M} \geq a, \quad-\eta \leq h_{\partial M} \leq \eta .
$$

for some strictly positive real numbers a and $\eta$. Setting $k^{\prime}=\min \{1, \beta\} \cdot k$, ( $\beta$ is the real number defined as $\beta=\inf _{\alpha \in \mathbb{R}^{+}}\left\{\alpha \geq \frac{1}{a \cdot k}\right\}$ ) then there exists a $C^{\infty}$ metric $\tilde{g}$ on $(M \sharp M, \tilde{g})$, isometric to $g$ in each copy of $M$ and such that

1. the sectional curvature has finite lower bound

$$
\sigma_{\tilde{g}} \geq-\left[k^{2}+\left(2 \eta+2 k^{\prime}+\frac{k^{2}}{\eta}\right) \sup \{\eta, k\}\right] .
$$

2. this metric is pinched, i.e.

$$
\begin{gathered}
\left(\frac{\sinh \left(\frac{k}{2 k^{\prime}}\right)}{\sinh \left(\frac{k}{k^{\prime}}\right)}\right)^{2} \cdot g \leq \tilde{g} \leq \\
{\left[\cosh \left(\frac{k}{2 k^{\prime}}\right)+\sup \left(1, \frac{\eta}{k}\right) \cdot \sinh \left(\frac{k}{k^{\prime}}\right)\right]^{2 n-2} \cdot\left(\frac{\sinh \left(\frac{k}{2 k^{\prime}}\right)}{\sinh \left(\frac{k}{k^{\prime}}\right)}\right)^{2 n-4} \cdot g .}
\end{gathered}
$$

Thanks to this theorem we get an estimate of the diameter of the doubled manifold which does not depend explicitly on the injectivity radius of the boundary. A direct consequence of the theorem is the estimate of the first non zero Laplacian eigenvalue and of the topological invariants of the manifold $(M, \partial M, g)$.

Estimates from above of the $p$-th eigenvalue are also calculated, extending a partial result for 2-dimensional manifolds of the writer (see $([9]), 2018)$ and using a previous result of Cheng.

\section{Definition of the doubled manifold $(M \sharp M, g \sharp g)$ and gen- eral properties of the spectrum.}

We summarize here how to build the "double Riemannian manifold $(M \sharp M, g \sharp g)$ " starting from a Riemannian manifold with non-empty boundary $(M, \partial M, g)$, the definition and the properties of the Laplacian and of its spectrum, referring to [8] to a complete and exhaustive analysis. The construction of a $C^{\infty}$ 
ESTIMATES OF THE LAPLACIAN SPECTRUM AND BOUNDS OF TOPOLOGICAL INVARIANTS FOR RIEMANNIAN MANIFOLDS WITH BOUNDARY II.

metric on the doubled manifold naturally arises, however its regularization controlling from below the sectional curvature is hard but unnecessary here, we assume indeed that the new metric $\tilde{g}$ on the doubled manifold $M \sharp M$ holds suitable properties, in this case also we refer to [8].

Let $(M, \partial M, g)$ be a Riemannian manifold with compact and differentiable boundary $\partial M$; from the disjoint union $M_{1} \amalg M_{2}$ of two copies of the manifold $M$ and the canonical maps $\psi_{1}$ and $\psi_{2}$ of $M$ on $M_{1}$ and $M_{2}$ we get the double $M \sharp M$ of $(M, \partial M)$ as the quotient manifold of $M_{1} \amalg M_{2}$ via the equivalence relation: $\psi_{1}(x) \sim \psi_{2}(x)$ if and only if $x \in \partial M$; in other words we define the doubled manifold as $(M \times\{1,-1\}) / \sim$, where the equivalence relation $\sim$ is defined as:

$$
(x, i) \sim(y, j) \text { if and only if }(x=y \text { and } i=j) \text { or }(x=y \in \partial M \text { and any } i, j)
$$

The two boundaries, that in this way are identified, yield a $(n-1)$-hypersurface named as "the equator" of $M \sharp M$. The manifold $M \sharp M$ can be equipped by a structure of $C^{\infty}$ manifold in the following way:

let $p:(M \times\{1,-1\}) \rightarrow M \sharp M$ be the canonical surjection, $U \subset p(\partial M \times$ $\{-1\})=p(\partial M \times\{1\})$ an open neighborhood in $M \sharp M$ and $N$ the $g$-unitary inward normal field of $\partial M$, the local chart $\Phi$ is defined as:

$$
\Phi(t, x)= \begin{cases}p\left(\exp _{x}[t \cdot N(x)], 1\right) & \text { if } \quad t \geq 0 \\ p\left(\exp _{x}[-t \cdot N(x)],-1\right) & \text { if } \quad t<0\end{cases}
$$

If $\varepsilon \leq \operatorname{inj}_{M}\left(\operatorname{inj}_{M}\right.$ the injectivity radius of $\left.M\right)$, the exponential normal map is a diffeomorphism of $] 0, \varepsilon[\times \partial M$ on its image in $M$ and the changes of charts are $C^{\infty}$-maps.

Let $j: M \rightarrow M \times\{1\}$ be the isometric immersion of $M$ in $M \times\{1\}$ and let $\Sigma: M \sharp M \rightarrow M \sharp M$ be the symmetry to respect the equator swapping the two copies of $M$ in $M \sharp M: \Sigma(M \times\{1\})=(M \times\{-1\})$. The map $j$ induces on $M \times\{1\}$ (resp. on $M \times\{-1\}$ ) the metric $g_{1}=j^{*}(g)$, (resp. the metric $\left.g_{-1}=\Sigma^{*}\left(g_{1}\right)\right)$. The passage to the quotient with respect the equivalent relation $\sim$ induces the metric $g \sharp g$ on $M \sharp M$.

Fact 2.1. The metric $g \sharp g$, as above defined on $M \sharp M$ is $C^{0}$ but not $C^{1}$; moreover it is a $C^{0}$-limit of $C^{\infty}$-metrics $g_{k}$ defined on $M \sharp M$.

Proof: See [8]. 
ESTIMATES OF THE LAPLACIAN SPECTRUM AND BOUNDS OF TOPOLOGICAL INVARIANTS FOR RIEMANNIAN MANIFOLDS WITH BOUNDARY II.

Let $(M, g)$ be a closed $C^{\infty}$ Riemannian manifold of dimension $n$, the metric $g$ and the Laplace operator write, in a local system of coordinates $\left(x^{1}, x^{2}, \ldots, x^{n}\right)$, respectively as $g=\sum_{i, j} g_{i j} d x^{i} \otimes d x^{j}$ and $\Delta=\sqrt{\operatorname{det} g^{-1}}$. $\frac{\partial}{\partial x^{i}}\left(\sqrt{\operatorname{det} g} \cdot g^{i j} \cdot \frac{\partial}{\partial x^{j}}\right)$; it is well known that the Laplacian is a self adjoint elliptic operator having a discrete sequence of positive eigenvalues going to infinity: $0 \leq \lambda_{0}<\lambda_{1} \leq \lambda_{2} \leq \cdots \leq \lambda_{i} \leq \cdots$. Moreover, each eigenspace $E\left(\lambda_{i}\right)$ has finite dimension, the direct sum of them is dense in $C^{\infty}(M)$ and the Hilbert space $L^{2}\left(M, d v_{g}\right)\left(d v_{g}\right.$ is the Riemannian measure on $\left.M\right)$ has a Hilbertian base of eigenfunctions.

Classic Lemma 2.2. For $C^{0}$-metrics on $(M \sharp M, g \sharp g)$ the spectrum of the Laplacian coincides with the critical values of the functional

$$
u \mapsto R(u)=\frac{\int_{M \sharp M}|d u|_{(g \sharp g)}^{2} d v_{(g \sharp g)}}{\int_{M \sharp M} u^{2} d v_{(g \sharp g)}}
$$

defined on $\mathcal{H}_{0}=H_{1}(M, g) \backslash\{0\}$. The critical points are calculated using the min-max principle or the max-min principle, i.e.

$$
\begin{aligned}
\lambda_{i}(M \sharp M, g \sharp g)= & \inf _{\mathcal{E}_{i+1}} \max _{u \in \mathcal{E} \backslash\{0\}} \frac{\int_{M \sharp M}|d u|_{g \sharp g}^{2} d v_{g \sharp g}}{\int_{M \sharp M} u^{2} d v_{g \sharp g}} \\
& =\sup _{\mathcal{E}_{i}} \inf _{u \in \mathcal{E}_{i}^{\perp} \backslash\{0\}} \frac{\int_{M \sharp M}|d u|_{g \sharp g}^{2} d v_{g \sharp g}}{\int_{M \sharp M} u^{2} d v_{(g \sharp g)}}
\end{aligned}
$$

being $\mathcal{E}_{i} \subset \mathcal{H}_{0}$ any vectorial subspace of dimension $i$ in $\mathcal{H}_{0}$.

Proof: See [2].

Lemma 2.3. Let $\left\{g_{k}\right\}_{k \in \mathbb{N}}$ be a sequence of $C^{\infty}$-metric converging in the $C^{0}$-topology to a $C^{0}$-limit metric on $M \sharp M$, then:

(i) $\operatorname{diam}(M \sharp M, g \sharp g)=\lim _{k \rightarrow+\infty} \operatorname{diam}\left(M \sharp M, g_{k}\right)$;

(ii) $\operatorname{Vol}(M \sharp M, g \sharp g)=\lim _{k \rightarrow+\infty} \operatorname{Vol}\left(M \sharp M, g_{k}\right)$;

(iii) $\lambda_{i}(M \sharp M, g \sharp g)=\lim _{k \rightarrow+\infty} \lambda_{i}\left(M \sharp M, g_{k}\right)$;

Proof: See [8].

Definition 2.4. A function $u \in C^{\infty}(M, \partial M, g)$ solves 
ESTIMATES OF THE LAPLACIAN SPECTRUM AND BOUNDS OF

TOPOLOGICAL INVARIANTS FOR RIEMANNIAN MANIFOLDS WITH

BOUNDARY II.

- the Dirichlet problem when

$$
\left\{\begin{array}{l}
\Delta u=0 \\
\left.u\right|_{\partial M}=0
\end{array}\right.
$$

- the Neumann problem when

$$
\left\{\begin{array}{l}
\Delta u=0 \\
\left.\frac{\partial u}{\partial N}\right|_{\partial M}=0
\end{array}\right.
$$

being $N$ the inward unit normal to the boundary $\partial M$.

Classic Lemma 2.5. Let $(M, \partial M, g)$ be a Riemannian manifold with nonempty boundary and $(M \sharp M, g \sharp g)$ its double; then

(i) the spectrum of $(M \sharp M, g \sharp g)$ is the union of the Dirichlet and of the Neumann spectrum of $(M, \partial M)$ :

$$
\left\{\lambda_{i}(M \sharp M, g \sharp g) \mid i \in \mathbb{N}\right\}=\left\{\lambda_{i}^{D}(M)_{i} \mid i \in \mathbb{N} \backslash\{0\}\right\} \cup\left\{\lambda_{i}^{N}(M)_{i} \mid i \in \mathbb{N}\right\}
$$

each eigenvalue has to be counted with its own multiplicity;

(ii) there exists a Hilbertian base of eigenfunctions such that the restriction to each copy of $M$ is an eigenfuction of Dirichlet or Neumann problem.

Proof: See $[8]$

We give here finally the following

Definition 2.6. A Riemmanian manifold $(M, \partial M, g)$ with non-empty boundary is said with non-convex boundary if the second fundamental form of the boundary $I I_{\partial M}$ is positive definite with respect to the inward normal $N$.

\section{Proof of the Main Theorem}

The construction of the new metric $\tilde{g}$ in the doubled manifold $M \sharp M$ and with the control from below of the sectional curvature is the same as in the Appendix of [8]; we send again to it for more details.

In what follows, if there is not ambiguity, we shall denote with $k$ the positive determination of $\sqrt{k^{2}}$. 
ESTIMATES OF THE LAPLACIAN SPECTRUM AND BOUNDS OF TOPOLOGICAL INVARIANTS FOR RIEMANNIAN MANIFOLDS WITH BOUNDARY II.

Lemma 3.1. Let $(M, \partial M, g)$ be a n-dimensional Riemannian manifold with pinched sectional curvature $-k^{2} \leq \sigma \leq k^{2}$ for some real $k$, with non-empty boundary $\partial M$ whose injectivity radius is greater than a strictly positive real constant a. Let $H_{t}=\{x \in M$ s.t. $d(x, \partial M)=t\}$ be the hyper-surface at $t-d i$ stance from the boundary $(t<a)$ and let $h_{t}$ be its second fundamental form (with respect to the inward normal). Running $x$ all over $H_{t}$, if

- $h_{\max }(t)$ is the maximum among the biggest eigenvalues of $h_{t}$ and

- $h_{m i n}(t)$ is the minimum among the lowest eigenvalues of $h_{t}$,

then, for every $t<\min \left\{a, \frac{1}{2 k}\right\}$ we get

$$
h_{\max }(t) \leq \sup \{\eta, k\} \quad \text { and } \quad h_{\min }(t) \geq-2 \eta-\frac{k^{2}}{\eta} .
$$

Proof: Let $x_{0} \in H_{t}$ be a point of the hyper-surface $H_{t}$ and let $\gamma$ be the geodesic passing in $x_{0}$ and normal to the boundary, parametrized with the arc length. For every Jacobi field $V$ along $\gamma$ and normal to it we have

$$
h_{\text {max }}(t, x)=\sup \frac{\left.h_{t}\right|_{x}(V, V)}{g(V, V)} .
$$

Let $\widetilde{V}$ the the Jacobi field that reaches the sup, we have

$$
h_{\max }(t, x)=\frac{g\left(D_{\dot{\gamma}} \widetilde{V}, \widetilde{V}\right)}{g(\widetilde{V}, \widetilde{V})} .
$$

Taking $\widetilde{V}$ such that $\|\widetilde{V}(0)\|=1$, we have

$$
\left\|\widetilde{V}^{\prime}(0)\right\|=\left\|D_{\dot{\gamma}} \widetilde{V}^{\prime}(0)\right\|=\eta \leq h_{\max }(0)
$$

To get a comparison we consider the corresponding Jacobi fields in the spaces of constant sectional curvature equal to $-k^{2}$ or $k^{2}$; these Jacobi fields will be denoted by a under-script 0 . If $\sigma \geq-k^{2}$, the Jacobi field writes

$$
\widetilde{V}_{0}(t)=\left(\frac{\eta}{k} \sinh k t+\cosh k t\right) v(t)
$$

being $v(t)$ the parallel transport of the vector $v(0)$ along $\gamma$ and such that $g_{0}(v(0), \dot{\gamma}(0))=0$. For the Rauch Comparison Theorem we get

$$
\frac{g\left(\widetilde{V}^{\prime}, \widetilde{V}\right)}{g(\widetilde{V}, \widetilde{V})} \leq \frac{g\left(\widetilde{V}_{0}^{\prime}, \widetilde{V}_{0}\right)}{g\left(\widetilde{V}_{0}, \widetilde{V}_{0}\right)}=\frac{\eta \cosh k t+k \sinh k t}{\frac{\eta}{k} \sinh k t+\cosh k t}=\frac{\eta+k \tanh k t}{1+\frac{\eta}{k} \tanh k t}
$$


ESTIMATES OF THE LAPLACIAN SPECTRUM AND BOUNDS OF

TOPOLOGICAL INVARIANTS FOR RIEMANNIAN MANIFOLDS WITH

BOUNDARY II.

which is an increasing function of $\eta$, it follows that

$$
h_{\max }(t, x) \leq k \cdot \frac{h_{\max }(0)+k \tanh k t}{k+h_{\max }(0) \tanh k t} .
$$

The function $t \mapsto h_{\max }$ is an increasing function of $t$ if $h_{\max }(0)<k$, we obtain

$$
h_{\max }(t) \leq \frac{h_{\max }(0)+k \tanh k t}{k+h_{\max }(0) \tanh k t} \cdot k \leq k ;
$$

however, if $h_{\max }(0)>k$ the function $t \mapsto h_{\max }$ is a decreasing function, getting

$$
h_{\max }(t) \frac{h_{\max }(0)+k \tanh k t}{k+h_{\max }(0) \tanh k t} \cdot k \leq \frac{h_{\max }(0)}{k} \cdot k=h_{\max }(0)=\eta .
$$

proving the upper bound of the second fundamental form of the hyper-surface $H_{t}$ at $t$ distance from the boundary, To get a lower bound of the second fundamental form of this hyper-surface we use as reference space the sphere of radius $k$; the related Jacobi field the field writes $\widetilde{V}_{0}(t)=\left(\frac{\eta}{k} \sin k t+\cos k t\right) v(t)$. For the Bishop Comparison Theorem we have

$$
\frac{g\left(\widetilde{V}^{\prime}, \widetilde{V}\right)}{g(\widetilde{V}, \widetilde{V})} \geq \frac{g\left(\widetilde{V}_{0}^{\prime}, \widetilde{V}_{0}\right)}{g\left(\widetilde{V}_{0}, \widetilde{V}_{0}\right)}=\frac{\eta-\tan k t}{\eta \tan k t+k} \cdot k
$$

this one is an increasing function of $\eta$ and a decreasing function of $t$. Taking $t<\frac{1}{k} \arctan \frac{k}{2 \eta}<\frac{1}{2 k}$ we obtain

$$
h_{\min }(t) \geq-2 \eta-\frac{k^{2}}{\eta} .
$$

which ends the proof of the Lemma.

We are able now to prove the two functional results of this paper collected in the above cited Main Theorem:

Proposition 3.2. Let $(M, \partial M, g)$ be a compact connected Riemannian manifold with non-empty and non-convex boundary such that the injectivity radius of the boundary has a non zero lower bound $a: \operatorname{inj}_{\partial M} \geq a>0$. Let $k$ and $\eta$ be two positive numbers such that

$$
-k^{2} \leq \sigma \leq k^{2} \quad-\eta \leq h_{\partial M} \leq \eta
$$


ESTIMATES OF THE LAPLACIAN SPECTRUM AND BOUNDS OF

Let $k^{\prime}=\min \{1, \beta\} \cdot k$ and $\beta$ the real number defined as $\beta=\inf _{\alpha \in \mathbb{R}^{+}}\{\alpha \geq$ $\left.\frac{1}{a \cdot k}\right\}$, then there exists a metric $\tilde{g}$ on the doubled manifold $(M \sharp M, \tilde{g})$, isometric to $g$ on each copy of $M$ and such that

$$
\tilde{\sigma} \geq-\left(k^{2}+\left(2 \eta+2 k^{\prime}+\frac{k^{2}}{\eta}\right) \sup \{\eta, k\}\right) .
$$

Proof: As in [8] we interpose a thin cylinder of length $l \leq \frac{1}{k^{\prime}}$ among the two copies of the manifold $(M, g)$, we regularize the inherited metric $g \sharp g$ via a $C^{\infty}$ function $\phi_{\frac{1}{2 k^{\prime}}}:[0,+\infty[\rightarrow[0,+\infty[$ in such a way to get estimates independent on the injectivity radius of the boundary. Let $\phi_{\frac{1}{2 k^{\prime}}}:[0,+\infty[\rightarrow$ $\left[0,+\infty\right.$ [ be this function such that $\phi_{\frac{1}{2 k^{\prime}}}^{\prime}\left(\frac{1}{k^{\prime}}\right)=1$ and thus $\int_{0}^{\frac{1}{k^{\prime}}} \phi_{\frac{1}{2 k^{\prime}}}{ }^{\prime \prime}(s) d s=$ 1. Its second derivative $\phi_{\frac{1}{2 k^{\prime}}}$ " has to satisfy the following properties:

- $\phi_{\frac{1}{2 k^{\prime}}}{ }^{\prime \prime} \in C^{\infty}([0,+\infty[)$;

- the support of $\phi_{\frac{1}{2 k^{\prime}}}$ ' is compact and also contained in the open $] 0, \frac{1}{k}[$;

- $\int_{0}^{\frac{1}{k}} \phi_{\frac{1}{2 k^{\prime}}}{ }^{\prime \prime}(t) d t=1$

- $0 \leq \phi_{\frac{1}{2 k^{\prime}}}{ }^{\prime \prime}(t) \leq 2 k^{\prime}$ and

- its graphic have to be symmetric with the right $x-\frac{1}{2 k^{\prime}}=0$.

It follows that $\phi_{\frac{1}{2 k^{\prime}}}^{\prime}\left(\frac{1}{k^{\prime}}-s\right)+\phi_{\frac{1}{2 k^{\prime}}}^{\prime}(s)=1$ and that $\int_{0}^{\frac{1}{k^{\prime}}} \phi_{\frac{1}{2 k^{\prime}}}^{\prime}(s) d s=\frac{1}{2 k^{\prime}}$; so $\phi_{\frac{1}{2 k^{\prime}}}(t)=\frac{1}{2 k^{\prime}}+\int_{0}^{t} \phi_{\frac{1}{2 k^{\prime}}}^{\prime}(s) d s$ remains. It follows that on $M \backslash M_{\frac{1}{2 k^{\prime}}}$ the metric $. \tilde{g}=d t^{2} \oplus g_{\phi(t)}$ is isometric to the metric $\frac{d t^{2}}{\left[\phi_{\frac{1}{2 k^{\prime}}}^{\prime}\left(\phi_{\frac{1}{2 k^{\prime}}}^{-1}(t)\right)\right]^{2}} \oplus g_{t}$. Moreover we emphasize here that, for every $t$, we have

$$
\begin{aligned}
& 0 \leq \quad \phi_{\frac{1}{2 k^{\prime}}}^{\prime}(t) \quad \leq 1 \\
& 0 \leq a(t)=\phi_{\frac{1}{2 k^{\prime}}}^{\prime 2 k^{\prime}}\left(\phi_{\frac{1}{2 \prime^{\prime}}}^{-1}(t)\right) \leq 1 \\
& 0 \leq a(t) \cdot q^{\prime}(t)=\phi_{\frac{1}{2 k^{\prime}}}^{\prime}{ }^{\prime}\left(\phi_{\frac{1}{2 k^{\prime}}}^{-1}(t)\right) \leq 2 k^{\prime} .
\end{aligned}
$$

Exploiting the results of Lemma 3.1 we get

$$
\begin{gathered}
\tilde{\sigma} \geq \sigma+\left(1-q^{2}(t)\right) h_{\max } h_{\min }-2 q(t) \cdot q^{\prime}(t) h_{\max } \geq \\
-\left(k^{2}+\left(2 \eta+2 k^{\prime}+\frac{k^{2}}{\eta}\right) \sup \{\eta, k\}\right) .
\end{gathered}
$$


ESTIMATES OF THE LAPLACIAN SPECTRUM AND BOUNDS OF

TOPOLOGICAL INVARIANTS FOR RIEMANNIAN MANIFOLDS WITH

BOUNDARY II.

which ends.

We get thus the estimations of the regularized metric of the doubled manifold thanks to the following

Proposition 3.3. With the same assumptions of the Proposition 3.2, there exists a $C^{\infty}$ metric $\tilde{g}$ on $M \sharp M$ such that

$$
\begin{gathered}
\left(\frac{\sinh \left(\frac{k}{2 k^{\prime}}\right)}{\sinh \left(\frac{k}{k^{\prime}}\right)}\right)^{2} \cdot g \leq \tilde{g} \leq \\
{\left[\cosh \left(\frac{k}{2 k^{\prime}}\right)+\sup \left(1, \frac{\eta}{k}\right) \cdot \sinh \left(\frac{k}{k^{\prime}}\right)\right]^{2 n-2} \cdot\left(\frac{\sinh \left(\frac{k}{2 k^{\prime}}\right)}{\sinh \left(\frac{k}{k^{\prime}}\right)}\right)^{2 n-4} \cdot g .}
\end{gathered}
$$

Proof: Let $\mathbb{J}$ be the set of all Jacobi fields normal to the geodesic $\gamma$, i.e. $J \in \mathbb{J} \Leftrightarrow g\left(J^{\prime}(t), \gamma^{\prime}(t)\right)=0$ for all $t$. At point $\gamma(t)=(x, t)$ we have

$$
\tilde{g} \geq \inf _{J \in \mathbb{J}} \frac{g(J(\phi(t)), J(\phi(t)))}{g(J(t), g(J(t))} \cdot g .
$$

Vector $J$ is indeed the image of a vector $X$, tangent to $\partial M$ via the tangent map to the chart $\Psi: \partial M \times] 0, a\left[\rightarrow M, \Psi(x, t)=\exp _{x}(t \cdot N(x))(N\right.$ is the $g$-unitary inward normal field of $\partial M)$. We consider the vector field $\bar{J}(t)=$ $\sinh [k(a-t)] \mathbf{u}, \mathbf{u}$ being a unit vector field normal to $\bar{\gamma}$ in the reference space of sectional curvature identically equal to $-k^{2}$. We get

$$
\begin{gathered}
\frac{g(J[\phi(t)], J[\phi(t)])}{g(J(t), J(t))} \geq \frac{g(J[\phi(t)], J[\phi(t)])}{\bar{g}(\bar{J}(t), \bar{J}(t))} \\
\geq \frac{\bar{g}(\bar{J}[\phi(t)], \bar{J}[\phi(t)])}{\bar{g}(\bar{J}(t), \bar{J}(t))} \geq \frac{\bar{J}^{2}(t+\varepsilon)}{\bar{J}^{2}(t)} \geq \frac{\bar{J}^{2}\left(\frac{1}{k^{\prime}}-\varepsilon\right)}{\bar{J}^{2}\left(\frac{1}{k^{\prime}}\right)},
\end{gathered}
$$

where the first inequality comes from Rauch Comparison Theorem, while the other ones derive from the fact that function $\frac{|J|}{\bar{J}}$ is a decreasing function. This chain of inequalities proves the left inequality of (ii). Let $\left\{\mu_{i}\right\}_{i=1, \ldots n}$ be the relative eigenvalues of $\tilde{g}$ to $g$; we have $\mu_{1}=1$ and $\mu_{i} \geq\left(\frac{\sinh \left[k\left(\frac{1}{k^{\prime}}-\varepsilon\right)\right]}{\sinh k\left(\frac{1}{k^{\prime}}\right)}\right)$ for $i \geq 2$. From what has just been proved in (i) we have

$$
\prod_{i=2}^{n} \mu_{i} \geq\left[\cosh (k t)+\frac{\eta}{\delta} \sinh (k t)\right]^{2 n-2} .
$$


ESTIMATES OF THE LAPLACIAN SPECTRUM AND BOUNDS OF TOPOLOGICAL INVARIANTS FOR RIEMANNIAN MANIFOLDS WITH BOUNDARY II.

This inequality shows an upper bound of each eigenvalue $\mu_{i}$ and consequently proves the inequalities chain in (ii). We conclude keeping $\varepsilon=\frac{1}{2 k^{\prime}}$, ending also the proof of the Main Theorem.

\section{Estimates of Laplacian Spectrum and of Betti Num- bers}

We are able now to extend the following

Theorem 4.1. (Payne and Weinberger (1957), [7]; Li and Yau (1980), [5]; Meyer (1986), [6]) Let $(M, g)$ be a $C^{\infty}$ compact Riemannian manifold of dimenision $n$, and $\delta$ and $D$ two strictly positive constants such that Ricci $_{g} \geq$ $-(n-1) \delta^{2} g$ and $\operatorname{diam}(M, g) \leq D$, then there exists a constant $C$, which depends only on the product $\delta \cdot D$, such that:

$$
\lambda_{1}(g) \cdot \operatorname{diam}^{2}(M, g) \geq C(\delta \cdot D) .
$$

Theorem 4.2. Let $D, a, \eta$ and $k$ be four strictly positive real numbers. For every compact connected $n$-dimensional Riemannian manifold $(M, \partial M, g)$ whose diameter is bounded from above by $D$ and the sectional curvature is pinched $-k^{2} \leq \sigma \leq k^{2}$, and such that its non-empty and non-convex boundary $\partial M$ has injectivity radius bounded from below from a, and the main curvature is pinched by $\eta$, i.e.

$\operatorname{diam}(M, g) \leq D, \quad-k^{2} \leq \sigma \leq k^{2}, \quad \operatorname{inj}_{\partial M} \geq a, \quad$ and $\quad-\eta \leq h_{\partial M} \leq \eta ;$ then there exists a constant $C^{\prime}$, depending on $n, D, k$ and $\eta$, such that

$$
\begin{gathered}
\lambda_{1}^{D}(M, g) \cdot \operatorname{diam}^{2}(M, g) \geq C^{\prime}\left(n, D, k^{\prime}, \eta\right) \\
\lambda_{1}^{N}(M, g) \cdot \operatorname{diam}^{2}(M, g) \geq C^{\prime}\left(n, D, k^{\prime}, \eta\right) .
\end{gathered}
$$

being $k^{\prime}=\min \{1, \beta\} \cdot k$ and $\beta$ a real number defined as above.

Proof: The manifold $M \sharp M$ is equipped with the regularized metric $\tilde{g}$ such that $\sigma_{\tilde{g}} \geq-\left(k^{2}+\left(2 \eta+2 k^{\prime}+\frac{k^{2}}{\eta}\right) \sup \{\eta, k\}\right)=-\Theta(k, \eta)$. We apply Theorem 4.1 to the compact manifold with empty boundary $(M \sharp M, \tilde{g})$, getting

$$
\lambda_{1}(M \sharp M, \tilde{g}) \cdot \operatorname{diam}^{2}(M \sharp M, \tilde{g}) \geq C(\Theta(K, \eta) \cdot D)
$$

and 
ESTIMATES OF THE LAPLACIAN SPECTRUM AND BOUNDS OF

$$
\begin{gathered}
\left.\lambda_{1}^{D}(M, g) \cdot \operatorname{diam}^{2}(M, g) \geq C(\Theta(K, \eta) \cdot D)\right) \\
\left.\lambda_{1}^{N}(M, g) \cdot \operatorname{diam}^{2}(M, g) \geq C(\Theta(K, \eta) \cdot D)\right) .
\end{gathered}
$$

where $C$ is the constant as in Theorem 4.1. For the sake of simplicity we call now

$u\left(k, k^{\prime}\right)=\left(\frac{\sinh \left(\frac{k}{k^{\prime}}\right)}{\sinh \left(\frac{k}{2 k^{\prime}}\right)}\right), \quad v\left(k, k^{\prime}, \eta\right)=\left[\cosh \left(\frac{k}{2 k^{\prime}}\right)+\sup \left(1, \frac{\eta}{k}\right) \cdot \sinh \left(\frac{k}{2 k^{\prime}}\right)\right]$

and, applying the Lemma 3.2, we obtain

$$
u\left(k, k^{\prime}\right)^{-2} \cdot g \leq \tilde{g} \leq v\left(k, k^{\prime}, \eta\right)^{2 n-2} u\left(k, k^{\prime}\right)^{2 n-4} \cdot g
$$

From the results of Proposition 2.3 and remember that, for pinched metrics $C_{1} \cdot g \leq \tilde{g} \leq C_{2} \cdot g\left(C_{1}\right.$ and $C_{2}$ are to strictly positive constants) we get

$$
C_{1}^{\frac{1}{2}} \cdot \operatorname{diam}(M, g) \leq \operatorname{diam}(M, \tilde{g}) \leq C_{2}^{\frac{1}{2}} \cdot \operatorname{diam}(M, g)
$$

and

$$
\frac{C_{1}^{\frac{n}{2}}}{C_{2}^{\frac{n}{2}+1}} \lambda_{i}(g) \leq \lambda(\tilde{g}) \leq \frac{C_{2}^{\frac{n}{2}}}{C_{1}^{\frac{n}{2}+1}} \lambda_{i}(g) .
$$

(se also the Classic Lemma 2.2), the following estimates naturally arise:

$$
\operatorname{diam}(M, \tilde{g}) \leq \operatorname{diam}(M, g) \cdot v\left(k, k^{\prime}, \eta\right)^{n-1} \cdot u\left(k, k^{\prime}\right)^{n-2}
$$

and

$$
\lambda_{1}(\tilde{g}) \leq v\left(k, k^{\prime}\right)^{n^{2}-n} u\left(k, k^{\prime}, \eta\right)^{n^{2}-n+2} \cdot \lambda_{1}(g) .
$$

Putting the previous inequalities in (4.1) we obtain

$$
\begin{aligned}
& \lambda_{1}^{D}(M \sharp M, \tilde{g}) \cdot \operatorname{diam}^{2}(M, g) \geq C^{\prime}\left(n, D, k, k^{\prime}, \eta\right) \\
& \lambda_{1}^{N}(M \sharp M, \tilde{g}) \cdot \operatorname{diam}^{2}(M, g) \geq C^{\prime}\left(n, D, k, k^{\prime}, \eta\right)
\end{aligned}
$$

being $C^{\prime}(n, D, k, a, \eta)=\frac{1}{4} C(\Theta(k, \eta) \cdot D) \cdot u\left(k, k^{\prime}\right)^{2-n^{2}-n} v\left(k, k^{\prime}, \eta\right)^{2-n^{2}-n}$.

The inequalities from 4.2 show the existence of the constant $C^{\prime}$ which depends on $n, D, k, k^{\prime}$ and $\eta$ as in the statement, and concurrently give a lower bound of the first non-zero eigenvalue of the Laplacian of the manifold 
ESTIMATES OF THE LAPLACIAN SPECTRUM AND BOUNDS OF

$(M, \partial M, g)$.

Estimates of the $p$-th eigenvalue of the Laplacian are available thanks to the results of Cheng (see [1], 1975) for compact $n$-dimensional Riemmanian manifolds with Ricci curvature bounded from below.

Theorem 4.3. (Cheng, (1976), [1]: Corollary 2.3) Let (M,g) be a n-dimensional Riemannian manifold with diameter $D$ and Ricci curvature bounded from below by $-(n-1) \delta^{2} \cdot g$, then

- when $n=2(m+1), m \in \mathbb{N} \cup\{0\}$

$$
\lambda_{p}(M, g) \leq \frac{2 m+1}{4} \delta+\frac{4 p^{2}\left(1+2^{m}\right)^{2} \pi^{2}}{D^{2}} ;
$$

- when $n=2 m+3, m \in \mathbb{N} \cup\{0\}$

$$
\lambda_{p}(M, g) \leq \frac{2 m+2}{4} \delta+\frac{4 p^{2}\left(1+2^{2 m}\right)^{2}\left(1+\pi^{2}\right)}{D^{2}} .
$$

The following Theorem extends the previous result:

Theorem 4.4. With the same assumptions of Theorem 4.2 the p-th eigenvalue is raised by:

- when $n=2(m+1), m \in \mathbb{N} \cup\{0\}$

$$
\begin{gathered}
\lambda_{p}(M \sharp M, \tilde{g}) \leq \\
{\left[v\left(k, k^{\prime}, \eta\right) u\left(k, k^{\prime}\right)\right]^{n-n^{2}} \cdot\left(\frac{2 m+1}{4} \delta+\frac{4 p^{2}\left(1+2^{m}\right)^{2} \pi^{2}}{D^{2}} u^{2}\left(k, k^{\prime}\right)\right) ;}
\end{gathered}
$$

- when $n=2 m+3, m \in \mathbb{N} \cup\{0\}$

$$
\begin{gathered}
\lambda_{p}(M \sharp M, \tilde{g}) \leq \\
{\left[v\left(k, k^{\prime}, \eta\right) u\left(k, k^{\prime}\right)\right]^{n-n^{2}} \cdot\left[\frac{2 m+2}{4} \delta+\frac{4 p^{2}\left(1+2^{2 m}\right)^{2}\left(1+\pi^{2}\right)}{D^{2}} u^{2}\left(k, k^{\prime}\right)\right] .}
\end{gathered}
$$

Proof: The proof is the same as in the Proposition 4.2 with the suitable modification of the diameter value given by the Proposition 3.3.

Estimates of the topologic invariants are given by the following 
ESTIMATES OF THE LAPLACIAN SPECTRUM AND BOUNDS OF TOPOLOGICAL INVARIANTS FOR RIEMANNIAN MANIFOLDS WITH BOUNDARY II.

Theorem 4.5. (Gromov (1981), [3]) Let $(M, g)$ be a compact connected $n$ dimensional Riemannian manifold, and $\delta$ and $D$ two positive numbers such that Ricci $_{g} \geq-\delta^{2}(n-1) g$ and $\operatorname{diam}_{g}(M) \leq D$, then there exists a constant $C$, which depends on the product $\delta \cdot D$ and on the dimension $n$, such that

$$
\operatorname{dim} H^{1}(M) \leq n \cdot \exp (n \delta \cdot D C(\delta \cdot D))
$$

Previous Theorem is so extended by the following

Theorem 4.6. With the same assumptions of the Theorem 4.2, we have:

$$
\operatorname{dim} H^{1}(M)+\operatorname{dim} H^{1}(M, \partial M) \leq n \cdot \exp \left(n \cdot C^{\prime}\left(n, D, k, k^{\prime}, \eta\right)\right)
$$

where $C^{\prime}(n, D, k, a, \eta)$ is a constant that depends only on $n, D, k, k^{\prime}, \eta$.

Proof: Remembering that $\operatorname{dim} H^{i}(M \sharp M)=\operatorname{dim} H^{i}(M, \partial M)+\operatorname{dim} H^{i}(M)$ (see [4] (2011)), the proof is the same as in Proposition 4.2. In this case the constant $C^{\prime}$, after the regularization of the metric, is given by

$$
C^{\prime}\left(n, D, k, k^{\prime}, \eta\right)=C\left[\Theta(k, \eta) \cdot D \cdot u\left(k, k^{\prime}\right)^{n-2} \cdot v\left(k, k^{\prime}, \eta\right)^{n-1}\right]
$$

where $C$ is the constant of Theorem 4.5 .

\section{References}

[1] S. Y. Cheng, Eigenvalue comparison theorems and geometrical applications, Math. Z. 143 (1975) 289-297.

[2] S. Gallot, D. Hulin, J. La Fontaine, Riemannian Geometry, Springer, Berlin (2007).

[3] M. Gromov: Structures métriques pour les variétés riemanniennes, Textes Math. 1, Cedic-Nathan, (1981).

[4] F. Laudenbach: A Morse Complex for Manifolds with boundary, Geom. Dedicata 153 (2011), pp. 47-57.

[5] Li, P., Yau, S.T.: Estimates of eigenvalues of a compact Riemannian manifold. Proc. Symp. Pure Appl. Math. 36, 205-239 (1980). 
ESTIMATES OF THE LAPLACIAN SPECTRUM AND BOUNDS OF

TOPOLOGICAL INVARIANTS FOR RIEMANNIAN MANIFOLDS WITH

BOUNDARY II.

[6] D. Meyer: Minoration de la premiere valeur propre non nulle du problème de Neumann sur les variétés riemanniens à bord, Ann. Inst. Fourier, Grenoble 36, 2 (1986), pp.113-125.

[7] L. E. Payne and H. F. Weinberger: Lower bounds for vibration frequencies of elastically supported membranes and plates, J. Soc. Ind. Appi. Math. 5 (1957), pp. 171-182.

[8] L. Sabatini: Estimates of the Laplacian Spectrum and bounds of Topological Invarinats for Riemannian Manifolds with Boundary, Analele Un. Ovidius Constanta, Seria Matematica, vol. XXVII fasc. 2, (2019), pp. 179-211.

[9] L. Sabatini: On the Vibration Frequencies of thin linear elastic Membranes, Applictions of Mathematics, vol. 63, (2018), pp. 3753.

[10] P. Yang, S. T. Yau: Eigenvalues of the Laplacian of compact Riemann surfaces and minimal submanifolds, Ann. Scuola N. Sup. Pisa 7 (1980), pp. 55-63. 